\title{
¿El entrenamiento en lectura de creencias puede influir sobre la competencia pragmática?: un estudio sobre las relaciones de estas capacidades cognitivas en la infancia
}

\author{
Ximena Restrepo; Johny Villada Zapata
}

Cómo citar este artículo:

Restrepo, X., \& Villada, J. (2021). ¿El entrenamiento en lectura de creencias puede influir sobre la competencia pragmática?: un estudio sobre las relaciones de estas capacidades cognitivas en la infancia. Acta Colombiana de Psicología, 24(2), 80-94. https://www.doi.org/10.14718/ACP.2021.24.2.8

Recibido, julio 22/2020; Concepto de evaluación, abril 08/2021; Aceptado, mayo 05/2021

\author{
Ximena Restrepo \\ ORCID: https://orcid.org/0000-0002-3942-0710 \\ Universidad de Antioquia, Medellín, Colombia. \\ Johny Villada Zapata \\ ORCID: https://orcid.org/0000-0002-9561-5344 \\ Universidad de Antioquia, Medellín, Colombia.
}

\begin{abstract}
Resumen
Con el objetivo de indagar respecto a la influencia de la lectura de creencias sobre la competencia pragmática, en el presente estudio 56 niños de entre 48 y 54 meses pertenecientes al programa Buen Comienzo de la ciudad de Medellín participaron en un diseño experimental pre-post con grupo control. Para la evaluación de la lectura de creencias se adaptaron al español cuatro pruebas de la escala de Wellman y Liu (2004), mientras que para evaluar la competencia pragmática se adaptaron cuatro situaciones comunicativas propuestas por Sacco et al. (2008). Para la manipulación de la variable se llevaron a cabo 10 sesiones de entrenamiento en lectura de creencias. De acuerdo con los resultados intragrupo, el programa de entrenamiento fue efectivo para mejorar la variable entrenada, pues el grupo experimental mejoró su desempeño en la lectura de creencias ( $p=.007 ; d=-.663)$ y en la competencia pragmática $(p=.007 ; d=.648)$; no obstante, aunque se encontraron diferencias significativas entre el grupo experimental y el grupo control para la lectura de creencias $(p=.001 ; d=.472)$, no fue así para el desempeño en la competencia pragmática $(p=.340 ; d=.143)$. A la luz de estos resultados, no es posible asegurar una relación de influencia de la lectura de creencias sobre la competencia pragmática; sin embargo, se encontró un modelo de regresión lineal satisfactorio cuando se incluyeron en los análisis variables sociodemográficas. Al final del artículo se discute respecto a la necesidad de integrar variables de contexto cuando se indaga por una posible relación de influencia de una variable psicológica sobre otra.

Palabras clave: teoría de la mente, competencia pragmática, niño en edad preescolar, entrenamiento.
\end{abstract}

\footnotetext{
Facultad de Ciencias Sociales y Humanas, Universidad de Antioquia, Medellín, Colombia. Calle 67 No 53-108 B1. 9 of. 227, C. P.: 050010.ximena.restrepol@udea.edu.co

El presente articulo se encuentra vinculado al trabajo de maestría "Relaciones entre la competencia pragmática y la lectura de creencias: efectos de dos programas de entrenamiento en niños de 4 años" (Restrepo, 2020) y a la tesis de grado "Relación entre Lectura de creencias y Competencia Pragmática y su evolución luego de un entrenamiento en Lectura de creencias” (Cerpa \& Yepes, 2020).
} 


\title{
Can belief- reading training influence pragmatic competence? A study on the relationships of these cognitive abilities in childhood
}

\begin{abstract}
In order to investigate the influence of belief-reading on pragmatic competence, 56 children between 48 and 54 months old, belonging to the program Buen Comienzo [Good Start] in the city of Medellín, participated in a pre-post experimental design with a control group. For the evaluation of belief-reading, four tests of the Wellman and Liu (2004) Scale were adapted to Spanish; while for the evaluation of pragmatic competence, four communicative situations proposed by Sacco et., al. (2008) were adapted. For the manipulation of the variable, 10 training sessions in belief-reading were carried out. According to the intragroup results, the training program was effective in improving the trained variable, as the experimental group improved its performance in belief reading $(\mathrm{p}=, 007 ; \mathrm{d}=-, 663)$ and pragmatic competence $(\mathrm{p}=, 007 ; \mathrm{d}=, 648)$. However, although significant differences were found between the experimental group and the control group for belief reading $(\mathrm{p}=, 001 ; \mathrm{d}=, 472)$, this was not the case for performance in pragmatic competence $(\mathrm{p}=, 340 ; \mathrm{d}=, 143)$. In light of these results, it is not possible to ensure a relationship of influence of belief reading on pragmatic competence. Nevertheless, a satisfactory linear regression model was found when sociodemographic variables were included in the analyzes. The need to integrate context variables when inquiring about a possible relationship of influence of one psychological variable on another is discussed.

Keywords: theory of mind, pragmatic competence, preschool children, training.
\end{abstract}

\section{Introducción}

La relación entre el lenguaje y la lectura de creencias (en adelante LC) ha ocupado gran parte de los esfuerzos en la investigación actual. A la fecha se han encontrado claras correlaciones estadísticas entre ambas capacidades a lo largo del ciclo vital tanto en población con desarrollo típico como atípico (Baixauli-Fortea et al., 2017; Deleau, 2012; Lecce et al., 2018); no obstante, Deleau (2012) argumenta que la correlación es insuficiente para entender si una de las variables es mejor predictora del desempeño que la otra. La discusión se hace más específica cuando se indaga cómo ocurre la relación entre la LC y las dimensiones del lenguaje — sintáctico, semántico y pragmáticoLa presente investigación se interesó, en particular, por la relación entre la LC y la dimensión pragmática del lenguaje.

En primer lugar, entendemos la competencia pragmática como la habilidad del hablante para comprender las palabras en contexto (Sperber \& Wilson, 2012). De acuerdo con Gutiérrez-Ordóñez (2005), esta competencia representa la habilidad para reconocer y usar información proveniente de lo explícito y lo implícito en las situaciones comunicativas; es decir, se trata de aquella capacidad que permite identificar la ubicación espaciotemporal de los mensajes, las relaciones entre el emisor y el receptor, la toma de turnos, y las normas de la interacción.

Por otra parte, comprendemos la LC como la capacidad para identificar que un sujeto tiene estados de representación que pueden o no corresponder con el mundo, y que dichos estados guían su comportamiento (Wellman, 2014). De acuerdo con Wellman y Liu (2004), existen cuatro logros cognitivos que permiten dicha capacidad: (a) tener acceso al conocimiento $(A C)$, es decir, la comprensión de que el conocimiento que tiene una persona acerca de un fenómeno está relacionado con el acceso perceptual al mismo — niños de tres años y medio pueden decir "ella no sabe qué estamos haciendo porque no nos está viendo"-; (b) contar con diversas creencias (DC), es decir, la comprensión de que pueden existir distintas perspectivas respecto a un mismo fenómeno - alrededor de los cuatro años un infante puede producir una frase del tipo "mi hermano cree que el balón está en el armario, pero yo digo que está en la caja"-; (c) identificar contenido de falsa creencia (CF), es decir, la comprensión de que un individuo puede confundirse entre lo que un objeto aparenta ser y lo que realmente es - este tipo de conocimiento es posible relacionarlo con la capacidad de los infantes para realizar bromas que utilizan la disonancia entre apariencia y realidad: "esto parece una caja de chocolates, pero en realidad tiene hormigas dentro", por ejemplo-; y (d) comprender una falsa creencia (FC), es decir, la comprensión del estado de representación errónea que tiene otro individuo sobre el estado real del mundo - "mi hermano cree que la muñeca está en la caja, pero yo sé dónde está escondida realmente", por ejemplo-

La evaluación de ambas capacidades en niños coincide en el uso de tareas experimentales en las que se les propone una situación comunicativa a los infantes por medio de viñetas o representaciones simuladas, y luego se les pide que respondan a preguntas que aluden a la comprensión de la situación o a la anticipación del comportamiento de los personajes.

Al respecto, Bermúdez (2009) plantea que para comprender las creencias son indispensables las habilidades en 
tareas verbales, $\mathrm{y}$, de hecho, se ha afirmado que algunas características particulares del lenguaje son necesarias para el desarrollo de la LC. Por ejemplo, Milligan et al. (2007) llevaron a cabo un metaanálisis para ahondar en dicha relación, y es posible agrupar sus conclusiones en dos afirmaciones: (a) para que los niños comprendan creencias es necesario que exista un mínimo de comprensión lingüística en dos vías - por un lado, comprender la complementariedad sintáctica, la cual hace posible que el infante entienda oraciones como "María piensa que o cree que", y, por otro, conocer el significado de los verbos relativos a estados mentales y creencias - ; y (b) aunque es posible identificar en artículos de discusión relaciones entre la dimensión pragmática del lenguaje y el desarrollo de la LC, hace falta un cuerpo más numeroso de trabajos que permita analizar la naturaleza de dicha relación.

Mendoza-Lara y López-Herrero (2004) coinciden con la segunda conclusión de Milligan et al. (2007), y afirman que la semántica y la sintaxis no explican completamente la manera en que el lenguaje permite a los niños resolver las tareas de falsa creencia; lo que quiere decir que el desarrollo de la semántica y la sintaxis son necesarias para que emerjan habilidades de comprensión de creencias, deseos e intenciones, pero no suficientes. Debido a esto, afirman, se hace necesario incluir en el análisis funciones pragmáticas referidas al intercambio comunicativo - como la atención conjunta y la toma de turnos-, pues estas constituyen un potente indicador del desarrollo posterior tanto de la capacidad mentalista como del lenguaje. De hecho, estos autores soportan su argumento en la evidencia de que las dificultades o imposibilidades para involucrarse en las actividades comunicativas con pares y adultos - p. ej., déficits o pérdida de la audición en la primera infancia-se relacionan con déficits para comprender las tareas de falsa creencia (Fujino et al., 2017; Jones et al., 2015).

En suma, diversos autores plantean que el lenguaje es el proceso cognitivo que favorece el desarrollo de la $\mathrm{LC}$ (Bermúdez, 2009; Bosco et al., 2018; Bosco \& Gabbatore, 2017; Deleau, 2012; Lecce et al., 2018), y, particularmente, que es la capacidad para comprender los mensajes, no solo en su contenido semántico y sintáctico, sino también en lo que significan pragmáticamente de acuerdo con la situación, lo que permite que los niños se desenvuelvan mejor en las tareas de LC (Westra, 2017; Westra \& Carruthers, 2017).

No obstante, la conclusión referida deja de lado una hipótesis alterna que no se ha descartado teórica ni empíricamente, a saber, que una mejor LC favorece el desempeño en tareas de pragmáticas. Esta hipótesis ha tenido lugar en tanto se ha encontrado que pacientes con traumas que afectan la comprensión de creencias presentan dificultades para comprender el significado no literal de sentencias de orden sarcástico e irónico. Al respecto, algunos autores afirman que comprender el estado mental de una persona, como las creencias, es clave para poder modificar el comportamiento del interlocutor por medio del uso del lenguaje y para lograr un efecto comunicativo particular (Bosco et al., 2017; Parola et al., 2018). Por su parte, Baixauli-Fortea et al. (2017) encontraron que un mejor desempeño en tareas mentalistas se encuentra relacionado con un mejor uso del contexto lingüístico en la conversación.

Estos antecedentes se vinculan con una visión del desarrollo del lenguaje en la que se entiende que para comprender los mensajes, los individuos necesitan reconocer lo que ocurre en el momento preciso de la interacción, es decir, quiénes son los hablantes y los objetos a los que se refieren y qué relación tienen entre ellos y con los objetos. En línea con esto, Belinchón et al. (2004) afirman que ser competentes en el lenguaje implica usar adecuadamente los significados de las palabras y adecuarlos no solo a la situación, sino también a los hablantes, lo que implica comprender que el interlocutor es un agente con deseos, intenciones y creencias, y que se comporta lingüísticamente en línea con dichos estados mentales.

A la luz de dichos antecedentes teóricos y empíricos, planteamos la pertinencia de indagar respecto al vínculo o la posible relación de influencia que tiene la capacidad de comprender estados mentales, particularmente las creencias, sobre la capacidad para comprender actos comunicativos pragmáticos.

Sumado a las propuestas que plantean una posible influencia de la lectura de creencias sobre la competencia pragmática, los antecedentes proponen una serie de variables moduladoras que, en conjunto, ayudan a explicar el posible efecto de la LC sobre la competencia pragmática. Por ejemplo, Poulin-Dubois (2020) señala que la riqueza de los intercambios conversacionales entre padres e hijos, hermanos y pares favorece el desarrollo de la comprensión de estados mentales y de intenciones comunicativas. En ese sentido, el nivel educativo de los padres puede estar relacionado con un contexto lingüístico más rico que puede ser ofrecido a los infantes (McMahon \& Bernier, 2017; Muhinyi \& Rowe, 2019; Zeegers et al., 2017), mientras que un contexto de negligencia o separación por parte de los padres podría afectar el desarrollo de habilidades de comprensión pragmática (Sante et al., 2020). De hecho, se ha documentado que vivir con pares - como hermanos o primos - facilita una mayor exposición a intercambios lingüísticos que exigen el dialogo sobre estados mentales e intenciones (Song \& Volling, 2018).

Así las cosas, para la presente investigación se propuso poner a prueba la hipótesis de la influencia de la LC sobre la competencia pragmática, considerando las siguientes 
variables posiblemente moduladoras: el nivel socioeconómico, el número de hermanos, el número de personas con quienes vive, y el nivel educativo de los padres.

Adicionalmente, como afirman Weimer et al. (2021), el lapso entre los cuatro y los cinco años representa un periodo de adquisición de un conjunto de habilidades que permiten la comprensión de las creencias en entornos comunicativos, así como un momento de transición en el que dicho conjunto de habilidades para comprender a los demás se complejiza; así, junto con los retos propios de los entornos educativos y familiares, puede entenderse este periodo como una ventana evolutiva que permite ver cambios significativos tanto en las habilidades lingüísticas como mentalistas. De este modo, entendemos que dicha ventana evolutiva da pie a la posibilidad de hacer manipulaciones a la variable y a la posibilidad de observar cambios en un periodo delimitado.

Finalmente, dado que el objetivo fue analizar cómo la mejora en la LC puede repercutir en una mejora en el desempeño en la competencia pragmática, diseñamos un estudio que permitiera medir ambas variables antes y después de la manipulación de la variable independiente. Para cumplir tal fin, utilizamos la estrategia de intervención de sesiones de entrenamiento, que consistió en actividades que enfatizaron las cuatro comprensiones mentalistas asociadas a las creencias descritas previamente, es decir, acceso al conocimiento (AC), diversas creencias (DC), contenido de falsa creencia $(\mathrm{CF})$, y falsa creencia $(\mathrm{FC})$.

\section{Método}

\section{Tipo de estudio y diseño}

Se realizó un estudio de corte empírico analítico, con un diseño experimental pre-post con grupo control (Ato et al., 2013). La muestra fue intencional, con asignación aleatoria a dos grupos: uno que recibió el entrenamiento (G1), y el otro, un grupo control (G2). Todos los participantes fueron evaluados en LC y en su competencia pragmática antes y después del tratamiento.

\section{Participantes}

La muestra final estuvo conformada por 56 participantes (33 niñas) de entre 48 y 54 meses, pertenecientes al programa Buen Comienzo de la ciudad de Medellín, Colombia, en dos centros: el Jardín infantil Moravia y el CI Coomulsap Horizontes. El $50 \%$ del total de la muestra fue asignado al grupo experimental, y el $50 \%$ restante al grupo control. Para la selección de los participantes se utilizó una base de datos de dos jardines de la ciudad de Medellín, de la cual se incluyeron niñas y niños en el rango de edad indicado con desarrollo típico, que dieron su consentimiento verbal para participar, y cuyos acudientes dieron su consentimiento de forma escrita. Se excluyeron de la muestra aquellos que no quisieron participar en las pruebas, aquellos que no pudieron ser evaluados en la fase postest de la investigación, y quienes no completaron la totalidad de las sesiones de entrenamiento. Adicionalmente, se excluyeron de la muestra los niños y niñas que obtuvieron puntuación máxima en ambas pruebas en la evaluación inicial.

\section{Instrumentos}

\section{Consentimiento informado y cuestionario sociodemográfico}

En el documento firmado por los acudientes se aclaró que los fines de la investigación fueron netamente académicos y que las actividades no implicaban riesgo para los niños. No se ofreció ningún tipo de remuneración por la participación de los niños y niñas. En el mismo documento, los acudientes accedieron a brindar información respecto a: número de semanas de gestación, enfermedades del participante, nivel socioeconómico, número de personas con quien vive el participante, número de hermanos $\mathrm{y}$ escolaridad de los padres.

\section{Tareas para la lectura de creencias}

Las tareas fueron adaptadas de la escala diseñada por Wellman y Liu (2004). Las cuatro tareas se tradujeron al español y se utilizaron imágenes propias para los objetos y los personajes de las historias (véase Tabla 1).

\section{Evaluación pragmática}

Se emplearon cuatro tareas de comprensión de actos comunicativos sinceros, inspiradas en Sacco et al. (2008), que consistían en situaciones comunicativas acompañadas de imágenes. La escala de calificación fue la siguiente: se otorgaba 1 punto a la respuesta adecuada al contexto, y 0 puntos a la respuesta inadecuada al contexto. Las láminas utilizadas en las situaciones fueron diseñadas con el sitio web Pixton (véase Tabla 2).

\section{Tareas de entrenamiento}

El diseño y evaluación de las sesiones estuvo orientado por la revisión de antecedentes (Hale \& Tager-Flusberg, 2003; Lecce et al., 2014; Lohmann \& Tomasello, 2003), de manera que se diseñaron de forma gradual de menor a mayor complejidad. Así, primero se presentaron las sesiones con actividades sobre el acceso al conocimiento, luego, actividades de comprensión de diversas creencias y comprensión de apariencia de la realidad en relación 
Tabla 1.

Tareas para la lectura de creencias

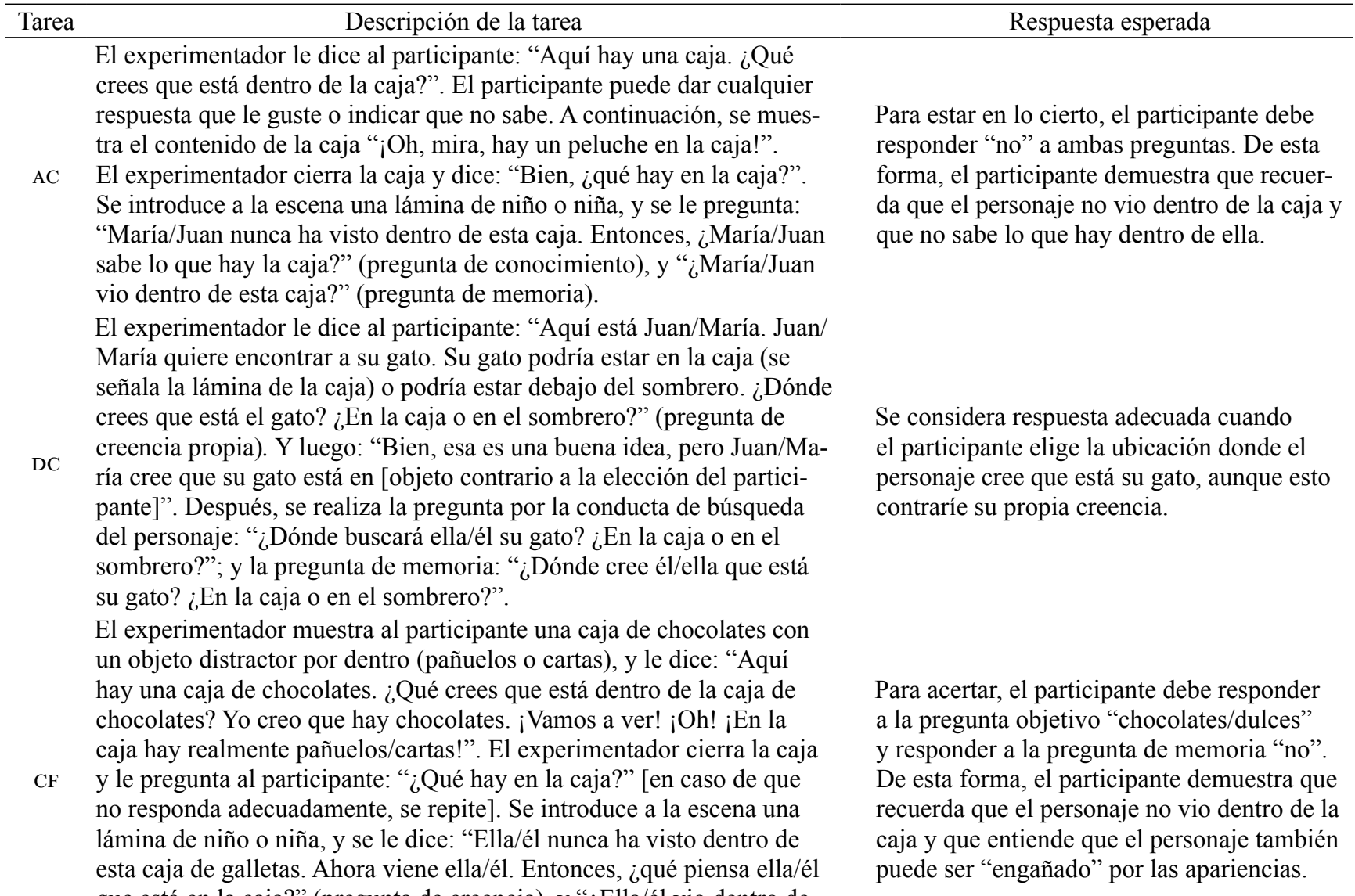

que está en la caja?” (pregunta de creencia), y “¿Ella/él vio dentro de esta caja?" (pregunta de memoria).

Se le muestra al participante una figura de juguete de un niño y una hoja de papel con un morral y un armario dibujados en ella, y se le dice: “Aquí está Juan, Juan quiere encontrar sus galletas. Sus galletas pueden estar en su morral o pueden estar en el armario". Se esconde la lámina del niño o niña. "Te voy a mostrar a ti, pero no a ella/él

FC dónde están las galletas. Realmente, las galletas están en el armario". Se introduce de nuevo la lámina del niño o niña. "Ella/él piensa que sus galletas están en su morral. Entonces, ¿dónde buscará ella/él sus galletas? ¿En su morral o en el armario?” (pregunta de conducta), y “¿Dónde están realmente las galletas de ella/él? ¿En su morral o en el armario?" (pregunta de memoria).

Para estar en lo cierto, el participante debe responder a la pregunta de conducta "morral" y responder a la pregunta de memoria "armario". De esta forma, el participante demuestra que recuerda la ubicación real del objeto y que entiende que el personaje buscará donde cree que está el objeto, no donde realmente está. con la tarea de contenido de falsa creencia, y, finalmente, actividades de entrenamiento en falsa creencia. Cada una de las sesiones de entrenamiento tuvo la siguiente estructura:

- Fase de familiarización: se iniciaba con un saludo, y junto a la profesora el experimentador proponía rondas infantiles propias del jardín. Esta fase permitía que la presencia del evaluador se hiciera familiar para el niño.

- Actividades de LC: actividades de manipulación de objetos — p. ej., imágenes impresas, juguetes, etc. - . Los participantes observaban un video o escuchaban una historia y el experimentador hacía preguntas que luego retroalimentaba verbalmente con las respuestas esperadas y explicaciones de estas. Así, durante la lectura de los cuentos e historias, se hizo énfasis en el contenido mentalista — qué saben los personajes, qué desconocen, si están en lo correcto o si están equivocados_- El experimentador hacía pausas para hacer preguntas asociadas a la memoria de los eventos, a las emociones de los personajes, $\mathrm{y}$ a los pensamientos. 
Tabla 2.

Tareas para la competencia pragmática

\begin{tabular}{|c|c|c|c|}
\hline Ítem & Descripción de la situación comunicativa & Respuesta esperada & Imagen \\
\hline 1 & $\begin{array}{l}\text { Camilo está en la mesa comiendo, mientras } \\
\text { que su madre está en la cocina. Camilo } \\
\text { dice: “Mmm, ¡Qué deliciosa está esta co- } \\
\text { mida! ¿Quién la hizo?” La madre responde: } \\
\text { “Sabía que lo iba a lograr!”. (a) ¿Qué } \\
\text { quiso decir la mamá? (b) ¿Quién hizo la } \\
\text { arepa? }\end{array}$ & $\begin{array}{l}\text { Se estima que el parti- } \\
\text { cipante comprende la } \\
\text { situación cuando puede } \\
\text { vincular la expresión } \\
\text { de la madre con que } \\
\text { fue ella quien realizó la } \\
\text { arepa y que sabía que le } \\
\text { iba a gustar. }\end{array}$ & \\
\hline 2 & $\begin{array}{l}\text { Felipe y María están en el sofá. Felipe se } \\
\text { acerca a la ventana y le muestra a María el } \\
\text { hermoso día soleado afuera. Felipe le pregun- } \\
\text { ta a María: “QQué quieres hacer hoy?”. (a) } \\
\text { ¿Qué podría decir la niña?. (En caso de duda: } \\
\text { ¿Qué significa eso?). }\end{array}$ & $\begin{array}{l}\text { Se estima que el par- } \\
\text { ticipante comprende } \\
\text { la situación cuando } \\
\text { infiere como posibles } \\
\text { actividades aquellas } \\
\text { que se relacionan con el } \\
\text { contexto comunicativo } \\
\text { que comparte Felipe con } \\
\text { María: día soleado. }\end{array}$ & \\
\hline 3 & $\begin{array}{l}\text { David está en la cocina y acaba de terminar } \\
\text { de cocinar. En la sala está su hermana. David } \\
\text { le muestra el plato, la llama con la cabeza (un } \\
\text { solo gesto hacia abajo, también acompañado } \\
\text { de un gesto con el brazo), como diciendo } \\
\text { “ ¿Vienes?”, y su hermana asiente (gesto } \\
\text { arriba abajo con la cabeza). (a) ¿Qué quería } \\
\text { decirle la niña al niño? (b) ¿La hermana va a } \\
\text { comer con David? }\end{array}$ & $\begin{array}{l}\text { Se estima que el par- } \\
\text { ticipante comprende } \\
\text { la situación gracias al } \\
\text { lenguaje no verbal. La } \\
\text { hermana acepta la comi- } \\
\text { da que se le ofrece. }\end{array}$ & \\
\hline 4 & $\begin{array}{l}\text { Pablo está estacionado al lado del camino. } \\
\text { Parece que lleva allí mucho tiempo porque } \\
\text { mira su reloj, se pone las manos por encima } \\
\text { de los ojos como buscando alguien que viene. } \\
\text { Cuando de repente aparece un carro. Pablo } \\
\text { necesita ayuda, y como los que vienen en el } \\
\text { carro no lo escuchan, empieza a hacer esto } \\
\text { con las manos (evaluador levanta las manos). } \\
\text { (a) ¿Qué está queriendo decir pablo? }\end{array}$ & $\begin{array}{l}\text { Se estima que el parti- } \\
\text { cipante comprende la } \\
\text { situación gracias al len- } \\
\text { guaje no verbal. Infiere } \\
\text { que Pablo pide ayuda. }\end{array}$ & \\
\hline
\end{tabular}

\section{Procedimiento}

Tanto las sesiones de evaluación como las de entrenamiento se realizaron en un aula del jardín, en la que se procuró tener condiciones de sonido y luz favorables. Para las fases pre y post se aplicaron las dos pruebas consecutivamente, y se aleatorizó su orden de presentación. Las sesiones de evaluación fueron grabadas para su posterior calificación y análisis, y su aplicación tomaba aproximadamente 10 minutos por participante.

Las sesiones de entrenamiento se realizaron con la presencia de una agente educativa informada con anterioridad respecto al objetivo de la investigación. La fase de entrenamiento se realizó en el lapso de un mes y consistió en 10 sesiones grupales. Cada grupo estuvo conformado por entre 3 y 7 participantes, y las sesiones duraron en promedio 15 minutos cada una. En los análisis se incluyeron aquellos estudiantes que participaron de todas las sesiones de entrenamiento sin falta, y se excluyeron aquellos que no respondieron de ninguna manera a las tareas o que se encontraban indispuestos en alguna de ellas.

\section{Consideraciones éticas}

Todos los procedimientos cumplieron con lo dispuesto en la Ley 1090 de 2006. El consentimiento de los acudientes fue una condición obligatoria para la participación de los infantes, y se tuvo en consideración el principio de beneficencia y no maleficencia. Finalmente, la presente investigación se comprometió a realizar una devolución a las instituciones y cuidadores en forma de conferencia. 


\section{Resultados}

En cuanto a los datos sociodemográficos, la muestra se distribuyó de la siguiente manera: la mayoría de los participantes pertenece a un nivel socioeconómico bajo y medio bajo (estratos 2 y $3^{1}$ ); el nivel de escolaridad de la mayoría de los padres y madres es de educación básica secundaria; con mayor frecuencia, los participantes conviven con cuatro o más personas; $y$, en su mayoría, los participantes son hijos únicos (véase Tabla 3).

Tabla 3.

\section{Tabla sociodemográficos}

\begin{tabular}{|c|c|c|c|}
\hline Variable & & Frecuencia & $\begin{array}{c}\text { Porcentaje } \\
(\%)\end{array}$ \\
\hline \multirow{3}{*}{ Estrato } & 1 & 4 & 7.143 \\
\hline & 2 & 23 & 41.071 \\
\hline & 3 & 22 & 39.286 \\
\hline \multirow{5}{*}{$\begin{array}{c}\text { Nivel de } \\
\text { escolaridad de la } \\
\text { madre }\end{array}$} & Primara & 2 & 3.571 \\
\hline & Secundaria & 23 & 41.071 \\
\hline & Técnico & 14 & 25.000 \\
\hline & Profesional & 9 & 16.071 \\
\hline & Especialización & 3 & 5.357 \\
\hline \multirow{5}{*}{$\begin{array}{c}\text { Nivel de } \\
\text { escolaridad del } \\
\text { padre }\end{array}$} & Primara & 9 & 16.071 \\
\hline & Secundaria & 20 & 35.714 \\
\hline & Técnico & 10 & 17.857 \\
\hline & Profesional & 3 & 5.357 \\
\hline & Especialización & 5 & 8.929 \\
\hline \multirow{3}{*}{$\begin{array}{l}\text { Número de } \\
\text { personas con } \\
\text { quien vive }\end{array}$} & 2 o menos & 16 & 28.571 \\
\hline & 3 personas & 12 & 21.429 \\
\hline & 4 o más & 23 & 41.071 \\
\hline \multirow{3}{*}{$\begin{array}{l}\text { Número de } \\
\text { hermanos }\end{array}$} & 0 & 24 & 42.857 \\
\hline & 1 & 19 & 33.929 \\
\hline & 2 o más & 8 & 14.286 \\
\hline
\end{tabular}

Para determinar el ajuste estadístico de los datos se utilizó la prueba Kolmogórov-Smirnov. Tras esto, se encontró que la muestra se distribuyó de forma no paramétrica tanto para la prueba de LC $(Z=0.00)$ como para la prueba

\footnotetext{
1 "La estratificación socioeconómica es la clasificación de los inmuebles residenciales de un municipio, que se hace en atención al Régimen de los Servicios Públicos Domiciliarios en Colombia (Ley 142 de 1994). [...] los estratos 1, 2 y 3 corresponden a estratos bajos que albergan a los usuarios con menores recursos, los cuales son beneficiarios de subsidios en los servicios públicos domiciliarios; los estratos 5 y 6 corresponden a estratos altos que albergan a los usuarios con mayores recursos económicos, los cuales deben pagar sobrecostos (contribución) sobre el valor de los servicios públicos domiciliarios” (DANE, 2021).
}

de competencia pragmática $(Z=0.00)$, de modo que se utilizaron pruebas no paramétricas para las comparaciones.

Se utilizó el estadístico Rho de Spearman para analizar la consistencia de las pruebas y subpruebas de pragmática y LC respecto al total, tras lo cual se encontró que los ítems de la prueba de pragmática correlacionan con el total, aunque no entre ellos — todas con valor $p<.001$ - así: ítem $1(r=.577)$, ítem $2(r=.463)$, ítem $3(r=.555)$, ítem $4(r=.542)$. Por tanto, concluimos que cada ítem evalúa aspectos distintos, aunque hacen parte del mismo constructo.

En cuanto a la prueba de LC, se encontraron correlaciones entre los ítems y la puntuación total - todas con valor $p>.05-$, así: AC $(r=.467), \mathrm{DC}(r=.505), \mathrm{CF}(r=.272), \mathrm{FC}$ $(r=.497)$. Adicionalmente, se encontró solo una correlación baja negativa entre DC y FC $(r=-.221, p=.043)$. En conclusión, encontramos consistente la prueba en LC, lo que quiere decir que evalúa un solo constructo dividido en distintos aspectos.

\section{Análisis intragrupo}

Con el fin de analizar posibles diferencias entre la evaluación pre y post por grupo, se utilizó la prueba de rangos con signo de Wilcoxon. Para esto, se tomaron en cuenta el valor $p$, y los tamaños del efecto (Cohen), interpretados a partir de los siguientes criterios: entre 0.20 y $0.40=$ pequeño; entre 0.41 y $0.60=$ moderado; y entre 0.61 y $1=$ grande.

El grupo entrenado en LC (grupo 1) mostró las siguientes diferencias para LC: en los ítems de AC, de CF, y en el total de LC, la media fue mayor en la evaluación post que en la evaluación pre (véase Tabla 4). En los tres casos el tamaño del efecto fue grande.

El grupo 1 también mejoró en la prueba de pragmática, así: en el ítem 1, 4, y en el total de la prueba de pragmática, la media fue mayor en la evaluación post que en la evaluación pre. Estas diferencias presentaron un tamaño del efecto grande. De acuerdo con estos resultados, podemos afirmar que el programa de entrenamiento en LC aporta mejoras al proceso de LC y al de pragmática (véase Tabla 5).

En el grupo control (grupo 2), respecto a la LC, no se encontraron diferencias entre la evaluación 1 y 2 . En línea con lo esperado, no se encontraron diferencias significativas en el desempeño en LC de los niños del grupo control; aunque sí se encontraron diferencias entre las medidas pretest y postest en los resultados de la prueba de pragmática para el grupo control: la media postest en los ítems 1 y 3 , y en el total de la prueba, fue mayor que la media de la evaluación pretest. En estos casos el tamaño del efecto fue grande. Dicho de otro modo, los niños que no recibieron ningún tipo de tratamiento mejoraron su desempeño entre la primera y la segunda evaluación de la prueba de pragmática por razones distintas al tratamiento (véase Tabla 6). 
Tabla 4.

Prueba de Wilcoxon para el grupo 1 en tareas de LC

\begin{tabular}{|c|c|c|c|c|c|c|c|c|c|}
\hline Variable & $\begin{array}{c}\text { Rango } \\
\text { promedio } \\
\text { positivo }\end{array}$ & $\begin{array}{c}\text { Suma de } \\
\text { rango } \\
\text { positivo }\end{array}$ & $\begin{array}{c}\text { Rango } \\
\text { promedio } \\
\text { negativo }\end{array}$ & $\begin{array}{c}\text { Suma de } \\
\text { rango } \\
\text { negativo }\end{array}$ & Media & Desviación & Z & Sig. & Cohen \\
\hline $\begin{array}{l}\text { AC pre } \\
\text { AC post }\end{array}$ & 6.50 & 78.00 & 0.00 & 0.00 & $\begin{array}{l}0.25 \\
0.68\end{array}$ & $\begin{array}{l}0.441 \\
0.476\end{array}$ & $-3.464^{b}$ & .001 & -1.000 \\
\hline $\begin{array}{l}\text { CF pre } \\
\text { CF post }\end{array}$ & 3.00 & 15.00 & 0.00 & 0.00 & $\begin{array}{l}0.04 \\
0.24\end{array}$ & $\begin{array}{l}0.200 \\
0.436\end{array}$ & $-2.236^{\mathrm{b}}$ & .025 & -1.000 \\
\hline $\begin{array}{l}\text { Total LC pre } \\
\text { Total LC post }\end{array}$ & 10.53 & 158.00 & 8.00 & 32.00 & $\begin{array}{l}1.25 \\
1.82 \\
\end{array}$ & $\begin{array}{l}0.752 \\
1.020 \\
\end{array}$ & $-2.694^{b}$ & .007 & -0.663 \\
\hline
\end{tabular}

Tabla 5.

Prueba de Wilcoxon para el grupo 1 en tareas de pragmática

\begin{tabular}{|c|c|c|c|c|c|c|c|c|c|}
\hline Variable & $\begin{array}{c}\text { Rango } \\
\text { promedio } \\
\text { positivo }\end{array}$ & $\begin{array}{c}\text { Suma de } \\
\text { rango } \\
\text { positivo }\end{array}$ & $\begin{array}{c}\text { Rango } \\
\text { promedio } \\
\text { negativo }\end{array}$ & $\begin{array}{c}\text { Suma de } \\
\text { rango } \\
\text { negativo }\end{array}$ & Media & Desviación & Z & Sig. & Cohen \\
\hline $\begin{array}{l}\text { Ítem } 1 \text { pre } \\
\text { Ítem } 1 \text { post }\end{array}$ & 6.00 & 54.00 & 6.00 & 12.00 & $\begin{array}{l}0.39 \\
0.64\end{array}$ & $\begin{array}{l}0.497 \\
0.488\end{array}$ & $-2.111^{\mathrm{b}}$ & .035 & -0.636 \\
\hline $\begin{array}{l}\text { Ítem } 4 \text { pre } \\
\text { Ítem } 4 \text { post }\end{array}$ & 5.50 & 49.50 & 5.50 & 5.50 & $\begin{array}{l}0.40 \\
0.72\end{array}$ & $\begin{array}{l}0.500 \\
0.458\end{array}$ & $-2.530^{\mathrm{b}}$ & .011 & -0.800 \\
\hline $\begin{array}{l}\text { Total pre } \\
\text { Total post }\end{array}$ & 13.03 & 208.50 & 7.42 & 44.50 & $\begin{array}{l}1.75 \\
2.64\end{array}$ & $\begin{array}{l}1.041 \\
1.193 \\
\end{array}$ & $-2.710^{\mathrm{b}}$ & .007 & -0.648 \\
\hline
\end{tabular}

Tabla 6.

Prueba de Wilcoxon para el grupo 2 en las tareas de pragmática

\begin{tabular}{cccccccccc}
\hline Variable & $\begin{array}{c}\text { Rango } \\
\text { promedio } \\
\text { positivo }\end{array}$ & $\begin{array}{c}\text { Suma de } \\
\text { rango } \\
\text { positivo }\end{array}$ & $\begin{array}{c}\text { Rango } \\
\text { promedio } \\
\text { negativo }\end{array}$ & $\begin{array}{c}\text { Suma de } \\
\text { rango } \\
\text { negativo }\end{array}$ & Media & Desviación & Z & Sig. & Cohen \\
\hline Ítem 1 pre & \multirow{2}{*}{7.50} & 97.50 & 7.50 & 7.50 & 0.16 & 0.374 & $-3.207^{\mathrm{b}}$ & .001 & -0.857 \\
Ítem1 post & & & & & 0.64 & 0.490 & & & \\
Ítem3 pre & \multirow{2}{*}{5.00} & \multirow{2}{*}{40.00} & 5.00 & 5.00 & 0.30 & 0.470 & $-2.333^{\mathrm{b}}$ & .020 & -0.778 \\
Ítem 3 post & & & & & 0.61 & 0.499 & & & \\
Total pre & \multirow{2}{*}{11.03} & \multirow{2}{*}{187.50} & \multirow{2}{*}{7.50} & 22.50 & 1.68 & 0.983 & $-3.220^{\mathrm{b}}$ & .001 & -0.786 \\
Total post & & & & & & & & & \\
\end{tabular}

\section{Análisis intergrupo}

Para comparar el desempeño de los grupos experimentales entre ellos y respecto al grupo control, y determinar posibles diferencias entre ellos en la evaluación posterior al tratamiento, se hizo uso de la prueba U de Mann-Whitney. Se utilizó esta misma prueba para analizar posibles diferencias por género, pero no se encontró ninguna diferencia.

\section{Desempeño en $L C$}

En cuanto al desempeño en LC, se encontraron diferencias entre el grupo control y el grupo experimental, así: (a) con un tamaño de efecto moderado, la media post de AC fue mayor para el grupo experimental respecto al control; (b) con un tamaño de efecto pequeño, la media del total de LC pre del grupo experimental fue mayor que la del grupo control; y (c) con un tamaño de efecto moderado, la media del total de LC post del grupo experimental fue mayor que la del grupo control. De este modo, los resultados indican que el programa de entrenamiento en LC generó una mejora en dicho proceso (véase Tabla 7).

Desempeño en la prueba de pragmática

En cuanto al desempeño en pragmática, se encontraron dos diferencias entre el grupo 1 y el grupo 2: (a) la media 
Tabla 7.

Comparación grupo 1 y 2 para las tareas de LC

\begin{tabular}{ccccccccccccc}
\hline Variable & $\begin{array}{c}\text { Media } \\
\text { G1 }\end{array}$ & DE G1 & $\begin{array}{c}\text { Media } \\
\text { G2 }\end{array}$ & $\begin{array}{c}\text { DE } \\
\text { G2 }\end{array}$ & $\begin{array}{c}\text { Rango } \\
\text { prome- } \\
\text { dio G1 }\end{array}$ & $\begin{array}{c}\text { Suma de } \\
\text { rangos } \\
\text { G1 }\end{array}$ & $\begin{array}{c}\text { Rango } \\
\text { promedio } \\
\text { G2 }\end{array}$ & $\begin{array}{c}\text { Suma de } \\
\text { rangos } \\
\text { G2 }\end{array}$ & $\begin{array}{c}\text { Ude } \\
\text { Mann- } \\
\text { Whitney }\end{array}$ & Z & Sig. & Cohen \\
\hline AC post & 0.68 & 0.476 & 0.21 & 0.418 & 35.00 & 980.00 & 22.00 & 616.00 & 210.000 & -3.463 & .001 & 0.464 \\
Total pre & 1.25 & 0.752 & 0.86 & 0.525 & 32.32 & 905.00 & 24.68 & 691.00 & 285.000 & -2.089 & .037 & 0.273 \\
Total post & 1.82 & 1.020 & 0.96 & 0.744 & 35.11 & 983.00 & 21.89 & 613.00 & 207.000 & -3.267 & .001 & 0.472 \\
\hline
\end{tabular}

Tabla 8.

Comparación entre los grupos 1 y 2 para las tareas de pragmática

\begin{tabular}{lcccccccccccc}
\hline Variable & $\begin{array}{c}\text { Media } \\
\text { G1 }\end{array}$ & $\begin{array}{c}D E \\
\text { G1 }\end{array}$ & $\begin{array}{c}\text { Media } \\
\text { G2 }\end{array}$ & $\begin{array}{c}\text { DE } \\
\text { G2 }\end{array}$ & $\begin{array}{c}\text { Rango } \\
\text { promedio } \\
\text { G1 }\end{array}$ & $\begin{array}{c}\text { Suma de } \\
\text { rangos } \\
\text { G1 }\end{array}$ & $\begin{array}{c}\text { Rango } \\
\text { promedio } \\
\text { G2 }\end{array}$ & $\begin{array}{c}\text { Suma de } \\
\text { rangos } \\
\text { G2 }\end{array}$ & $\begin{array}{c}\text { Ude } \\
\text { Mann- } \\
\text { Whitney }\end{array}$ & Z & Sig. & Cohen \\
\hline Ítem 1 pre & 0.39 & 0.497 & 0.15 & 0.362 & 31.30 & 876.50 & 24.57 & 663.50 & 285.500 & -2.019 & .044 & 0.245 \\
Ítem 4 pre & 0.37 & 0.492 & 0.68 & 0.476 & 23.69 & 639.50 & 32.16 & 900.50 & 261.500 & -2.268 & .023 & -0.308 \\
Total post & 2.64 & 1.193 & 2.39 & 1.031 & 30.50 & 854.00 & 26.50 & 742.00 & 336.000 & -0.954 & .340 & 0.143 \\
\hline
\end{tabular}

del ítem 1 de la prueba pre fue mayor para el grupo 1; y (b) la media del ítem 4 de la prueba pre fue mayor para el grupo 2 (véase Tabla 8). No se encontró ninguna diferencia respecto al total. De este modo, se puede afirmar que las diferencias encontradas en la evaluación pre no pueden ser explicados por los programas de entrenamiento.

\section{Modelos}

Para analizar si era posible identificar algunas variables sociodemográficas como predictoras del desempeño en pragmática diferenciando por grupos se llevaron a cabo regresiones lineales con el método hacia atrás (BackWard). De acuerdo con el objetivo de la presente investigación, para el análisis solo se utilizaron los datos post, es decir, cuando la variable ya había sido manipulada.

En general, se encontró un modelo estadísticamente satisfactorio en el grupo 1, pero no para el grupo 2. Al tomar el desempeño en pragmática post como variable dependiente y las siguientes como variables predictoras - total LC post, número de hermanos, nivel escolaridad de la madre, sexo, nivel socioeconómico (estrato), AC post, FC post, nivel escolaridad del padre, CF post, y número de personas con quienes vive el niño o niña-, se encontró un modelo que explica el $81 \%$ de la varianza (véase Tabla 9).
Por otra parte, de acuerdo con los valores beta, la variable que mejor contribuyó al modelo fue el estrato, y la que menos varianza aportó fue el sexo (véase Tabla 10).

\section{Discusión}

De acuerdo con los resultados, quienes recibieron entrenamiento en LC mejoraron su desempeño en algunos aspectos de la LC y en su desempeño para comprender actos comunicativos pragmáticos. Las mejoras en LC para el grupo 1 se presentaron en la comprensión del acceso al conocimiento y en el total de la escala de evaluación de LC, mientras que las mejoras en pragmática ocurrieron en el total de la prueba y en dos de las situaciones comunicativas propuestas: en el ítem 1, que evaluaba la capacidad del participante para entender una expresión sincera pero ambigua del lenguaje al vincular una expresión previa de uno de los emisores con elementos del contexto - esto es, que fue la madre quien realizó la arepa y quien sabía que le iba a gustar a su hijo-; y en el ítem 4, que evaluaba la comprensión del participante de la situación gracias al lenguaje no verbal — al inferir que Pablo pide ayuda por los elementos que presenta la situación-. No obstante, no se encontraron diferencias en el desempeño en la competencia

Tabla 9.

Coeficientes de determinación para el modelo predictivo de pragmática post en el grupo 1

\begin{tabular}{|c|c|c|c|c|c|c|}
\hline Modelo & $R$ & $R^{2}$ & $R^{2}$ Ajustado & SEE & $F$ & $p$ \\
\hline $\begin{array}{l}\text { (constante), total LC post, \# hermanos, nivel escolaridad } \\
\text { madre, sexo, estrato, AC post, FC post, nivel escolaridad } \\
\text { padre, CF post, \# personas con quienes vive }\end{array}$ & .901 & .812 & .604 & 0.643 & 3.893 & $.026 \mathrm{c}$ \\
\hline
\end{tabular}


Tabla 10.

Coeficientes de la regresión múltiple para pragmática post en el grupo 1

\begin{tabular}{|c|c|c|c|c|c|c|}
\hline \multirow{2}{*}{ Grupo } & \multirow{2}{*}{ Modelo } & \multicolumn{2}{|c|}{ Coeficientes no estandarizados } & \multirow{2}{*}{$\begin{array}{c}\text { Coeficientes estandarizados } \\
\text { B }\end{array}$} & \multirow[b]{2}{*}{$t$} & \multirow[b]{2}{*}{$p$} \\
\hline & & Beta & $\mathrm{EE}$ & & & \\
\hline \multirow{11}{*}{1} & (Constante) & 0.254 & 1.234 & & 0.206 & .841 \\
\hline & Sexo & 0.100 & 0.321 & 0.050 & 0.311 & .763 \\
\hline & Estrato & 1.366 & 0.399 & 0.673 & 3.428 & .008 \\
\hline & \# personas con quienes vive & -0.203 & 0.157 & -0.479 & -1.291 & .229 \\
\hline & \# hermanos & -0.103 & 0.142 & -0.224 & -0.727 & .486 \\
\hline & Nivel escolaridad del padre & -0.347 & 0.214 & -0.364 & -1.620 & .140 \\
\hline & Nivel escolaridad de la madre & 0.362 & 0.210 & 0.333 & 1.723 & .119 \\
\hline & AC post & -0.293 & 0.442 & -0.135 & -0.662 & .525 \\
\hline & CF post & 1.038 & 0.716 & 0.478 & 1.449 & .181 \\
\hline & FC post & 0.346 & 0.485 & 0.173 & 0.714 & .493 \\
\hline & Total LC post & -0.200 & 0.397 & -0.195 & -0.503 & .627 \\
\hline
\end{tabular}

pragmática entre el grupo experimental y el grupo control. Con el fin de analizar tales resultados, revisaremos críticamente nuestro programa de entrenamiento en su efecto para el grupo 1 por sí solo, y luego comparado con el grupo control.

Ahora bien, en las sesiones de entrenamiento en LC se enfatizó en las diversas perspectivas - lo que es sabido o desconocido por un agente- la posible confusión entre la apariencia y la realidad que puede tener un personaje, y la disonancia entre el estado del mundo y el estado de creencia de un agente respecto al mundo. Este entrenamiento se orientó en conjunto hacia la comprensión de las creencias y buscó no enfatizar en alguna variable específica dentro de la LC. No obstante, aunque se encontraron mejoras en el total de la prueba en LC, se resalta que, en particular, el acceso al conocimiento fue la capacidad que mostró mayores mejoras. Al respecto, es posible que en las situaciones de interacción social la información del acceso perceptual de los agentes involucrados en el intercambio comunicativo tenga más saliencia y pueda ser integrada con mayor facilidad.

Además de las actividades específicas sobre acceso al conocimiento llevadas a cabo en el entrenamiento, al inicio de cada sesión se realizaron actividades de familiarización que remarcaban estados de conocimiento e ignorancia de los agentes de las historias. En adición, antes de realizar las preguntas sobre las creencias, se insistía en el aspecto perceptual involucrado en el nivel de conocimiento de un agente respecto a un objeto, conocimiento del cual dependía el nivel de ajuste de la creencia al estado real del mundo. Por ejemplo, se preguntaba a los participantes: "Cuéntame, ¿que ve el personaje en este momento?, ¿el personaje vio cuando la rana se cambió de ubicación?". Gracias a esto, podemos afirmar que resaltar la información perceptual de las situaciones comunicativas facilita la comprensión de las creencias. Asimismo, en línea con Wellman (2014), afirmamos que el acceso al conocimiento es una habilidad central en la comprensión de creencias, y que dicha habilidad, aunque puede ser entendida como una capacidad independiente, es andamio de habilidades más complejas de comprensión de estados mentales, como lo es la falsa creencia.

Por otra parte, es posible establecer un vínculo entre la mejora en LC, el acceso al conocimiento, y las mejoras presentadas por el grupo 1 en dos situaciones comunicativas pragmáticas. Tomemos por ejemplo uno de los ítems que presentó mejoras: la situación comunicativa 1. Para responder a esta situación, el participante debía comprender que la expresión de la madre "sabía que lo iba a lograr" estaba vinculada con la expresión previa del niño en la mesa. Además, debía comprender que la atención de la madre estaba dirigida al niño. Si el participante era capaz de atender a tal información, podía inferir que lo que la madre quiso lograr fue que la arepa le quedara deliciosa.

En este punto, Woensdregt y Smith (2016) plantean una clara relación entre la competencia pragmática y la comprensión del acceso al conocimiento; de acuerdo con ellos, es necesario un nivel de razonamiento sobre la mente de los otros para ser competentes en lo pragmático del lenguaje. Específicamente, los autores proponen que el hablante tiene que, primero, tener presente el conocimiento sobre la situación comunicativa compartida con su interlocutor con el fin de seleccionar la información relevante para comprender el mensaje, y, segundo, comportarse lingüísticamente de forma adecuada con las circunstancias de la conversación. La primera condición planteada por los autores recuerda la conceptualización del acceso al conocimiento, es decir, que comprender lo que los otros quieren 
decir es facilitado por la capacidad para reconocer el estado de conocimiento que un agente tiene al respecto de cierto estado de cosas compartido por los interlocutores. Así, si el hablante identifica que su interlocutor desconoce algo de la situación conversacional, puede inferir que con su mensaje se está refiriendo solo a aquello que conoce de la situación. En este sentido, de acuerdo con los resultados del grupo experimental, planteamos hasta aquí que la comprensión del acceso al conocimiento es una capacidad que facilita la comprensión de los actos comunicativos pragmáticos, y, en esa medida, que una mejora en la primera puede favorecer un mejor desempeño en la segunda.

Ahora bien, cuando analizamos los resultados del grupo experimental comparados con el grupo control, encontramos que la mejora en la habilidad pragmática no es estadísticamente significativa respecto a la mejora del grupo que no recibió entrenamiento. De hecho, el grupo control no mejoró en ningún aspecto de LC, aunque mejoró en dos situaciones comunicativas pragmáticas y en el total de la prueba. A la luz de tal resultado, consideramos que es necesario ser cuidadosos en afirmar algo sobre sobre la influencia de LC respecto a la competencia pragmática, desde cuando esto no implique negar una relación entre dichas variables.

De otro lado, podemos afirmar que la comprensión del AC puede favorecer una mejor comprensión de situaciones comunicativas, pues integrar la información acerca del acceso perceptual que tienen o no los interlocutores con el fin de identificar las intenciones de estos últimos le permite al infante comprender con mayor facilidad las situaciones comunicativas pragmáticas. Sin embargo, la información del acceso perceptual del interlocutor no es la única información que puede permitir la comprensión un mensaje. Volvamos a las dos condiciones planteadas por Woensdregt y Smith (2016): la primera vincula el AC con la comprensión efectiva del significado de un mensaje, mientras que la segunda refiere a un aspecto paralelo a la comprensión mentalista, esto es, lo que es apropiado en determinada situación. Para comprender el significado de los mensajes, un agente tiene a disposición, a su vez, lo que su interlocutor sabe o desconoce, y el repertorio de situaciones familiares - las memorias de cómo él u otros han respondido a tales situaciones-.

En suma, proponemos que la comprensión de los actos comunicativos no es un asunto de todo o nada. Para comunicarse, los individuos disponen de herramientas cognitivas y utilizan las más eficientes para resolver las brechas de la comunicación - p. ej., la capacidad para inhibir información irrelevante o la memoria acerca de lo que se dijo previamente en la situación-. Además, la comprensión de los mensajes es un proceso de ensayo y error que, de acuerdo con las habilidades de las que disponga el individuo, su historial de aprendizaje — e incluso la habilidad comunicativa del interlocutor-puede resultar mayor o menor. Por tanto, concluimos que la relación entre la LC y la competencia pragmática no es necesariamente estable a través de los contextos comunicativos, ya que cada contexto comunicativo exige demandas diferenciables en cuanto al razonamiento que se requiere para comprender el significado de los mensajes.

Ahora bien, es posible que una mejor LC favorezca el desempeño situaciones comunicativas más opacos semánticamente, con un menor número de pistas contextuales, o en las cuales el contexto se encuentre menos estructurado - p. ej., cuando la tarea requiere alta demanda ejecutiva, como cuando se le pide al sujeto que responda sobre situaciones hipotéticas en las cuales no hay un referente directo de los hablantes-. En cambio, tareas con contextos bien estructurados pueden ser resueltas adecuadamente a partir de las pistas de tiempo y lugar, y no del estado mental del interlocutor, incluso a pesar de déficits en la LC.

Aunque nuestros resultados no nos permiten garantizar contundentemente la influencia de la LC sobre la competencia pragmática, sí podemos afirmar que ambas capacidades se encuentran relacionadas, y que juntas permiten a los individuos desenvolverse en un amplio margen de situaciones comunicativas. Así, en línea con Belinchón et al. (2004), planteamos que para evaluar las intenciones y necesidades informativas del interlocutor es requerida una serie de desarrollos en la comprensión de la mente de los demás tanto en el conocimiento léxico como en el morfosintáctico, aunque ninguna de estas habilidades por separado garantiza el éxito en la comprensión de los actos comunicativos. De este modo, comprender la mente de quien produce el mensaje es fundamental en las situaciones comunicativas.

De hecho, los mensajes tienen un significado por sí solos - aquello que significan intratextualmente-, y a la vez son producidos y escuchados por agentes con mente. Al respecto, Belinchón et al. (2004) afirman que la comprensión de los mensajes no puede ser garantizada por un mejor desempeño solo en uno de los conocimientos que soportan la competencia pragmática. En este sentido, nos apoyamos en una visión de la comunicación en la cual los individuos intentan comprender el significado de los mensajes lo mejor que pueden, y para ello hacen uso de las herramientas cognitivas y de la información de la que disponen, pero no necesariamente de todo ello a la vez, sino de aquellos elementos que resultan ser relevantes para la situación.

Basados en los resultados expuestos hasta aquí, si bien pudimos rastrear relaciones entre las variables de estudio, la 
posible influencia de la LC sobre la competencia pragmática aún es poco sólida, puesto que el grupo entrenado en LC, aunque mejoró entre la primera y la segunda evaluación de la habilidad pragmática, no lo hizo significativamente en comparación con el grupo control. No obstante, cuando planteamos un modelo que además de la influencia de la variable psicológica LC incluye otras variables sociodemográficas, se encuentra que es posible hablar de una relación predictiva de la LC respecto al desempeño de la habilidad pragmática en el grupo experimental.

Específicamente, en el modelo de regresión lineal vinculamos el total de LC, el AC, el contenido de falsa creencia y la falsa creencia con las siguientes variables: número de hermanos, nivel escolaridad de la madre y del padre, sexo, nivel socioeconómico, y número de personas con quienes vive el niño - cabe resaltar que el modelo explica un $81 \%$ de la varianza - . Analicemos primero cómo las variables predictoras se relacionaron entre sí, y luego cómo pueden estar relacionadas con la competencia pragmática.

Serrano (2013) afirma que el desarrollo de la LC es posible, entre otras variables, gracias a la influencia de factores del contexto familiar y social del niño. Por tanto, es esperable que la escolaridad de la madre y el padre sean buenos predictores de una mejor comprensión de creencias (Shin et al., 2020), ya que se ha afirmado que un mayor nivel educativo de los padres puede brindar un contexto semántico enriquecido al niño. Incluso, Ruffman et al. (2002) afirman que si la figura cuidadora utiliza regularmente verbos mentalistas en la interacción con el infante, esto puede favorecer el desarrollo de la comprensión de estados mentales como las creencias.

En línea con lo anterior, Dunn et al. (1991) afirman que tal efecto de exposición se produce igualmente con el uso de verbos que aluden a sentimientos de los miembros de la familia durante los juegos cooperativos con los hermanos o con sus pares. Adicionalmente, Ruffman et at. (1999) encontraron que el tiempo que las madres pasaban con los niños implementando estrategias disciplinarias de reflexión sobre los sentimientos de los demás se relacionaba positivamente con una mejor comprensión de creencias, comparados con quienes pasaban poco tiempo con sus madres y cuyas madres no incentivaban la reflexión sobre los sentimientos de las otras personas. Finalmente, Ruffman et at. (2018) reafirman dichos resultados al constatar que los padres que establecen conversaciones con sus hijos sobre estados mentales con mayor frecuencia favorecen un mejor desarrollo de la teoría de la mente en los niños. Los resultados de dichos autores soportan la idea de que las relaciones entre padres e hijos son importantes para la comprensión de las creencias.
Ahora bien, otra de las variables asociadas al desempeño en lectura de creencias es la conformación del núcleo familiar, pues el número de hermanos y la posición que se ocupa entre hermanos ha resultado ser una variable que permite predecir el desempeño de los niños en tareas mentalistas. De hecho, se ha encontrado que participar en actividades cooperativas o competitivas con hermanos o pares favorece experiencias discursivas enriquecidas respecto a los pensamientos y emociones de sí mismos y de los otros (Jenkins \& Astington, 1996; Peterson, 2000). En este sentido, Perner et al. (1994) encontraron que tener una familia numerosa se relaciona con mejores resultados en tareas que implicaban identificar cuándo un personaje tenía una falsa creencia, en comparación con los resultados de niños con familias pequeñas. Por su parte, Ruffman et al. (1998) encontraron que tener al menos un hermano mayor favorece el desarrollo de la comprensión de estados mentalistas; los autores explican que, a través del juego de simulación y el uso de verbos que aluden a los estados mentales - como a emociones propias y ajenas-, los niños pueden hacerse paulatinamente más hábiles para identificar los estados mentales de otras personas. En adición, Prime et al. (2016) encontraron que la influencia de los hermanos en el desarrollo de la teoría de la mente no se debe solo al número, sino a la sensibilidad cognitiva que presenten ellos, es decir, que tener hermanos mayores que comprenden mejor los estados mentales de los demás puede favorecer el desarrollo de la teoría de la mente de los hermanos menores.

Además, se ha discutido cómo la variable género modula el desempeño en LC. De acuerdo con Wacker et al. (2017), distintas investigaciones han encontrado con mayor recurrencia que las niñas se desempeñan mejor en tareas de comprensión mentalista. En la búsqueda de explicación de tales resultados, se ha indagado respecto a posibles diferencias biológicas o de carácter evolutivo, por ejemplo, de cómo los roles y la división del trabajo "moldeó" de forma diferente el cerebro de hombres y mujeres. Aun bajo este panorama, la discusión está abierta, y resultados contradictorios han encontrado una ventaja para el género masculino cuando se utilizan tareas que utilizan viñetas (véase Russell et al., 2007). Aunque en el presente estudio no se encontró ningún efecto por género, esta sí resulto ser una variable predictora dentro del modelo.

Por otra parte, podemos afirmar que el modelo encontrado pone de manifiesto cómo factores extrapsicológicos se encuentran vinculados a la capacidad para comprender estados mentales. Así, si se indaga por la influencia de la LC sobre la competencia pragmática, es necesario entender cómo la capacidad mentalista se encuentra influenciada por factores del contexto social del niño. En este sentido, una 
mejor LC puede favorecer un mejor desempeño pragmático cuando existen condiciones contextuales favorables; por ejemplo, desarrollarse en un entorno discursivo rico en verbos mentales, acompañado de pares y hermanos más capaces lingüísticamente que sirvan al infante como interpretes mentalistas y que le ayuden a comprender lo opaco del mensaje por medio de comprender la mente de quien habla.

En conjunto, los resultados de la presente investigación permiten aportar a la discusión respecto a cómo estudiar las relaciones entre habilidades de orden cognitivo. Queda claro que el intento por comprender relaciones entre habilidades por sí solas, sin entender el entramado de relaciones y procesos sociales que tienen lugar en el desarrollo de los niños, es insuficiente. Ahora, aunque el modelo de regresión lineal encontrado da luces sobre qué variables se relacionan con la LC y a su vez permite predecir el desempeño en la competencia pragmática, aún falta comprender los nexos dinámico-causales que explican las relaciones entre las variables encontradas en el modelo, y si además de las variables encontradas otras más pueden estar involucradas.

No obstante, si bien nuestra investigación no alcanza para comprender a profundidad cómo se relacionan las variables encontradas en el modelo, no es arbitrario que aparecieran dichas variables y no otras, como lo es el nivel de escolaridad de los padres y el número de hermanos. Es por esto que encontramos necesario integrar a los hallazgos empíricos propuestas teóricas de un autor clásico en psicología que comprende el fenómeno del desarrollo como un desarrollo complejo no lineal. Estamos de acuerdo con Vygotsky (1931/2012) cuando afirma que indagar en las relaciones humanas que anteceden y acompañan el desarrollo de los niños es necesario para comprender cómo ellos logran hacer propios los signos e interiorizar el significado y uso de las palabras - p. ej., las palabras que se refieren a estados mentales, tales como creer, desear, conocer-.

En conclusión, las interacciones comunicativas con pares pueden ofrecer variadas y enriquecidas formas de relacionarse lingüísticamente con el mundo. Lo que los resultados de la presente investigación sugieren es que la pregunta por si la lectura de creencias influye en la comprensión de los niños en las situaciones comunicativas pragmáticas puede entenderse de la mano con una visión en la cual el desarrollo de los procesos psicológicos es posible gracias a un entramado de factores tanto internos como externos. En palabras de Vygotsky (1931/2012), entendemos el desarrollo como:

[...] un complejo proceso dialéctico que se distingue por una complicada periodicidad, la desproporción en el desarrollo de las diversas funciones, las metamorfosis o transformación cualitativa de unas formas en otras, un entrelazamiento complejo de procesos evolutivos e involutivos, el complejo cruce de factores externos e internos, un complejo proceso de superación de dificultades y de adaptación (p. 141).

\section{Referencias}

Ato, M., López, J., \& Benavente, A. (2013). Un sistema de clasificación de los diseños de investigación en psicología. Anales de Psicología, 29(3), 1038-1059, http://dx.doi. org/10.6018/analesps.29.3.178511

Baixauli-Fortea, I., Casas, M., Berenguer-Forner, A. ColomerDiago, C., \& Roselló-Miranda, B. (2017). Pragmatic competence of children with autism spectrum disorder. Impact of theory of mind, verbal working memory, ADHD symptoms, and structural language. Applied Neuropsychology: Child, 8(2), 101-112. https://doi.org/10.1080/21622965.20 17.1392861

Belinchón, M., Igoa, J., \& Rivière, A. (2004). Psicología del lenguaje. Investigación y teoría (6. ${ }^{\mathrm{a}} \mathrm{ed}$.). Trotta S. A.

Bermúdez, M. (2009). Teorías infantiles de la mente y lenguaje: ¿un problema de huevo o gallina? Revista Iberoamericana de Psicología, 2(1), 67-85. http://dx.doi.org/10.33881/20271786.rip. 2209

Bosco, F. M., \& Gabbatore, I. (2017). Sincere, Deceitful, and Ironic Communicative Acts and the Role of the Theory of Mind in Childhood. Frontiers in Psychology, 8(21). https:// doi.org/10.3389/fpsyg.2017.00021

Bosco, F. M., Parola, A., Sacco, K., Zettin, M., \& Angeleri, R. (2017). Communicative-pragmatic disorders in traumatic brain injury: The role of theory of mind and executive functions. Brain and Language, 168, 73-83. https://doi. org/10.1016/j.bandl.2017.01.007

Bosco, F. M., Tirassa, M., \& Gabbatore, I. (2018). Why pragmatics and Theory of Mind do not (Completely) overlap. Frontiers in Psychology, 9, 1-7. https://doi.org/10.3389/ fpsyg.2018.01453

Cerpa, L., \& Yepes, E. (2020). Relación entre Lectura de creencias y Competencia Pragmática y su evolución luego de un entrenamiento en Lectura de creencias [Tesis de pregrado no publicada]. Universidad de Antioquia.

Departamento Administrativo Nacional de Estadística [DANE]. (15 de abril de 2021). Estratificación socioeconómica. https:// www.dane.gov.co/index.php/servicios-al-ciudadano/116espanol/informacion-georreferenciada/2421-estratifica cion-socioeconomica-preguntas-frecuentes\#: :text=La\%20 ESTRATIFICACI\%C3\%93N\%20SOCIOECON\%C3\%93 MICA\%20es\%20la,(Ley\%20142\%20de\%201994)

Deleau, M. (2012). Language and theory of mind: Why pragmatics matter. European Journal of Developmental Psychology, 9(3), 295-312. https://doi.org/10.1080/174056 29.2012.680303 
Dunn, J., Brown, J., \& Beardsall, L. (1991). Family talk about feeling states and children's later understanding of others' emotions. Developmental Psychology, 27(3), 448-455. https://doi.org/10.1037/0012-1649.27.3.448

Fujino, H., Fukushima, K., \& Fujiyoshi, A. (2017). Theory of mind and language development in Japanese children with hearing loss. International Journal of Pediatric Otorhinolaryngology, 96, 77-83. https://doi.org/10.1016/j. ijporl.2017.03.005

Gutiérrez-Ordóñez, S. (22-25 de septiembre de 2005). Ejercitarás la competencia pragmática. XVI Congreso Internacional de la ASELE: la Competencia Pragmática o la Enseñanza del Español como Lengua Extranjera. Oviedo. https://cvc.cervantes.es/ensenanza/biblioteca_ele/asele/ pdf/16/16_0025.pdf

Hale, C. M., \& Tager-Flusberg, H. (2003). The influence of language on theory of mind: a training study. Developmental Science, 6(3), 346-359. https://doi. org/10.1111/1467-7687.00289

Jenkins, J. M., \& Astington, J. (1996). Cognitive factors and family structure associated with theory of mind development in young children. Developmental Psychology, 32(1), 70-78. https://doi.org/10.1037/0012-1649.32.1.70

Jones, A. C., Gutierrez, R., \& Ludlow, A. K. (2015). Confronting the language barrier: Theory of mind in deaf children. Journal of Communication Disorders, 56, 47-58. https:// doi.org/10.1016/j.jcomdis.2015.06.005

Lecce, S., Bianco, F., Demicheli, P., \& Cavallini, E. (2014). Training Preschoolers on First-Order False Belief Understanding: Transfer on Advanced TdM Skills and Metamemory. Child Development, 85(6), 2404-2418. https://doi.org/10.1111/cdev.12267

Lecce, S., Ronchi, L., Del Sette, P., Bischetti, L., \& Bambini, V. (2018). Interpreting physical and mental metaphors: Is Theory of Mind associated with pragmatics in middle childhood? Journal of Child Language, 46(2), 393-407. https:// doi.org/10.1017/s030500091800048x

Lohmann, H., \& Tomasello, M. (2003). The Role of Language in the Development of False Belief Understanding: A Training Study. Child Development, 74(4), 1130-1144. https://doi.org/10.1111/1467-8624.00597

McMahon, C., \& Bernier, A. (2017). Twenty years of research on parental mind-mindedness: Empirical findings, theoretical and methodological challenges, and new directions. Developmental Review, 46, 54-80. https://doi.org/10.1016/j. dr.2017.07.001

Mendoza-Lara, E., \& López-Herrero, P. (2004). Consideraciones sobre el desarrollo de la teoría de la mente (TdM) y el lenguaje. Revista de Psicología General y Aplicada, 57(1), 4967. https://dialnet.unirioja.es/descarga/articulo/856428.pdf

Milligan, K., Astington, J., \& Dack, L. (2007). Language and Theory of Mind: Meta-Analysis of the Relation Between Language Ability and False-belief Understanding.
Child Development, 78(2), 622-646. https://doi. org/10.1111/j.1467-8624.2007.01018.x

Muhinyi, A., \& Rowe, M. (2019). Shared reading with preverbal infants and later language development. Journal of Applied Developmental Psychology, 64, 101053. https:// doi.org/10.1016/j.appdev.2019.101053

Parola, A., Berardinelli, L., \& Bosco, F. M. (2018). Cognitive abilities and theory of mind in explaining communicative-pragmatic disorders in patients with schizophrenia. Psychiatry Research, 260, 144-151. https://doi. org/10.1016/j.psychres.2017.11.051

Perner, J., Ruffman, T., \& Leekman, S. R. (1994). Theory of mind is contagious: You catch it from four sibs. Child Development, 65(4), 1228-1238. https://doi. org/10.1111/j.1467-8624.1994.tb00814.x

Peterson, C. C. (2000). Kindred spirits: Influences of siblings' perspectives on theory of mind. Cognitive Development, 15(4), 435-455. https://doi.org/10.1016/ S0885-2014(01)00040

Poulin-Dubois, D. (2020). Theory of mind development: state of the science and future directions. Progress in Brain Research, 254, 141-166. https://doi.org/10.1016/ bs.pbr.2020.05.021

Prime, H., Plamondon, A., Pauker, S., Perlman, M., \& Jenkins, J. M. (2016). Sibling cognitive sensitivity as a moderator of the relationship between sibship size and children's theory of mind: a longitudinal analysis. Cognitive Development, 39(July-September), 93-102. https://doi.org/10.1016/j. cogdev.2016.03.005

Restrepo, X. (2020). Relaciones entre la competencia pragmática y la lectura de creencias: efectos de dos programas de entrenamiento en niños de 4 años [Tesis de maestría no publicada]. Universidad de Antioquia.

Ruffman, T., Slade, L., \& Crowe, E. (2002). The Relation between Children's and Mothers Mental State Language and Theory-of-Mind Understanding. Child Development, 73(3), 734-751. https://doi.org/10.1111/1467-8624.00435

Ruffman, T., Perner, J., \& Parkin, L. (1998). Older (but not younger) siblings facilitate false belief understanding. Developmental Psychology, 34(1), 161-74. https://doi. org/10.1037/0012-1649.34.1.161

Ruffman, T., Perner, J., \& Parkin, L. (1999). How parenting style affects false belief understanding. Social Development, 8(3), 395-411. https://doi.org/10.1111/1467-9507.00103

Ruffman, T., Puri, A., Galloway, O., Su, J., \& Taumoepeau, M. (2018). Variety in parental use of "want" relates to subsequent growth in children's theory of mind. Developmental Psychology, 54(4), 677-688. https://doi.org/10.1037/ dev0000459

Russell, T. A., Tchanturia, K., Rahman, Q., \& Schmidt, U. (2007). Sex differences in theory of mind: A male advantage on Happé's “cartoon” task. Cognition y Emotion, 21(7), 1554-1564. https://doi.org/10.1080/02699930601117096 
Sacco, K., Angeleri, R., Bosco, F., Colle L., Mate, D., \& Bara, B. (2008). Assessment Battery for Communication-ABaCo: A new instrument for the evaluation of pragmatic abilities. Journal of Cognitive Science, 9(2), 111-157. https://pdfs. semanticscholar.org/4c40/ee5e22f35b95b3d71e35c79db36f4cbe65a6.pdf

Sante, M., Sylvestre, A., Bouchard, C., \& Leblond, J. (2020). Parental behaviors associated with the level of pragmatic language ability among 42-month-old neglected children. Child Abuse \& Neglect, 104, 104482. https://doi. org/10.1016/j.chiabu.2020.104482

Serrano, J. (2013). Desarrollo de la teoría de la mente, lenguaje y funciones ejecutivas en niños de 4 a 12 años [Tesis doctoral]. Universidad de Girona, España. http://hdl.handle. net/10803/123549

Shin, S., Leech, K., \& Rowe, M. (2020). Examining relations between parent-child narrative talk and children's episodic foresight and theory of mind. Cognitive Development, 55, 100910. https://doi.org/10.1016/j.cogdev.2020.100910

Song, J., \& Volling, B. (2018). Theory-of-Mind Development and Early Sibling Relationships after the Birth of a Sibling: Parental Discipline Matters. Infant \& Child Development, 27(1), e2053. https://doi.org/10.1002/icd.2053

Sperber, D., \& Wilson, D. (2012). Pragmatics, modularity and mindreading. Meaning and Relevance, 17, 261-278. https:// doi.org/10.1017/CBO9781139028370.016

Vigotsky, L. (1931/2012). El problema del desarrollo de las funciones psíquicas superiores. En Obras escogidas (Tomo 3). Machado Nuevo Aprendizaje.

Wacker, R., Bölte, S., \& Dziobek, I. (2017). Women Know Better What Other Women Think and Feel: Gender Effects on Mindreading across the Adult Life Span. Frontiers in Psychology, 8, Article 1324. https://doi.org/10.3389/ fpsyg.2017.01324

Weimer, A., Rice, K., Ettekal, I., Cartwright, K., Guajardo, N., \& Liew, J. (2021). Correlates and antecedents of theory of mind development during middle childhood and adolescence: An integrated model. Developmental Review, 59, Article 100945. https://doi.org/10.1016/j.dr.2020.100945

Wellman, H. M. (2014). Making minds: How theory of mind develops. Oxford University Press.

Wellman, H. M., \& Liu, D. (2004). Scaling of theory-of-mind tasks. Child Development, 75(2), 523-541. https://doi. org/10.1111/j.1467-8624.2004.00691.x

Westra, E. (2017). Pragmatic Development and the False Belief Task. Review of Philosophy and Psychology, 8(2), 235-257. https://doi.org/10.1007/s13164-016-0320-5

Westra, E., \& Carruthers, P. (2017). Pragmatic development explains the Theory-of-Mind Scale. Cognition, 158, 165-176. https://doi.org/10.1016/j.cognition.2016.10.021

Woensdregt, M., \& Smith, K. (2016). Pragmatics and Language Evolution. Oxford Research Encyclopedia of Linguistics. https://doi.org/10.1093/acrefore/9780199384655.013.321

Zeegers, M., Colonnesi, C., Stam, G., \& Meins, E. (2017). Mind matters: A three-level meta-analysis on parental mentalization and sensitivity as predictors of infant-Parent attachment. Psychological Bulletin, 143(12), 1245-1272. https://doi.org/10.1037/bul0000114 